\title{
Does Certification of an Arthroplasty Center according to Endocert Improve Quality or Complication Rate in Total Hip Arthroplasty in a General Hospital? Don't Follow Germany!
}

\author{
Lohani Bibek $^{1,2}$, Wolfgang Hönle ${ }^{2}$, Stefan Sesselmann ${ }^{3}$, Alexander Scheller ${ }^{2}$, Philipp Köhl ${ }^{4}$, \\ Alexander Schuh ${ }^{5 *}$ \\ ${ }^{1}$ Martha-Maria Hospital Nürnberg, 90491 Nürnberg, Germany \\ ${ }^{2}$ Musculoskeletal Center, Neumarkt Hospital, 92318 Neumarkt, Germany \\ ${ }^{3}$ Institute for Medical Technology, Ostbayerische Technische Hochschule Amberg-Weiden, Hetzenrichter Weg \\ 15, 92637, Weiden, Germany \\ ${ }^{4}$ Hospital of trauma surgery, Marktredwitz Hospital, 95615 Marktredwitz, Deutschland \\ ${ }^{5}$ Department of musculoskeletal research, Marktredwitz Hospital, 95615 Marktredwitz, Germany
}

*Corresponding Author: Prof. Dr. med. Alexander Schuh, Department of Musculoskeletal Research, Marktredwitz Hospital, 95615 Marktredwitz, Germany, Email: A.Schuh@Klinikum-Fichtelgebirge.de

\begin{abstract}
Question

Center of endoprothesis (EPZ) was established in Germany in 2012 to improve quality of TKA or THA. Aim of the current study is to investigate, whether there are any changes in quality or incidence of complications in total hip arthroplasty (THA) by establishing a center of endoprothesis (EPZ) in a general hospital especially in respect to so called quality parameters.
\end{abstract}

\begin{abstract}
Material and Methods
We conducted a retrospevtive study comparing the first 100 THA's one year before establishing of an EPZ (Group I) with the first 100 THA's one year after establishing an EPZ (group II). Data were collected by analyzing our electronic documentation system, and report of the rehabilitation hospital. Especially so called quality parameters according to EndoCert were considered. Statistic analysis was performed with SPSS 22.0. using Kolmogorow-Smirnow-Test and Mann-Whitney U Test and chi-square-test.
\end{abstract}

\section{Results}

$X$-rays before and after THA were available in all cases in group I and II ( $p>0,05)$.

Preoperative planning was performed in all cases in group I and group II ( $p><0,05)$.

In group I we found 5 complications, in group II were 4 complications documented $(p>0,05)$. In group I we found 2 cases with an inclination angle $>50^{\circ}$, in group II in one case ( $\left.>>0,05\right)$. Mean duration of THA was 74.0 minutes in group I (min: 35, max: 175) and 70.0 minutes in group II (min: 30, max: 120) $(p>0,05)$. Operation time above 90 minutes was found in 19/100 cases (19\%) in group and in 13/100 cases (13\%) in group I $(p<0,001)$.

\section{Conclusion}

Establishing a center of endoprosthesis (EPZ) in a general hospital leads to few positive changes. Only parameter "duration of operation $>100$ minutes showed a significant improvement. This slight improvement of quality does not justify high personal and financial expenses. We believe that quality management systems are necessary in THA or TKR but should include clinical relevant parameters and should not be such expensive and time demanding. Future studies will show whether other certification systems like DIN EN ISO9001:2015 are enough. At that moment we recommend not to follow this german concept.

Keywords: Total Hip Arthroplasty, EPZ, Quality, Complications 


\section{INTRODUCTION}

Total hip arthroplasty (THA) was named the operation of the $20^{\text {th }}$ century due to its great clinical outcome [1,2]. A shorter hospital stay and lower medical costs has been shown for high volume centers and established aftercare standards [3, 4]. To improve Quality in THA so called Arthroplasty Centers were established in Germany in 2012. Certification of arthroplasty centers according to EndoCert (EPZ or EPZ Max) has asserted itself rapidly and there are meanwhile more than 400 certificated centres in Germany $[4,5]$. The recognition of the quality indicators and their implemantation are regulated by EndoCert since 2012 [2, 5, 6]. A certification can be obtained by fulfilling a number of institutional requirements, and sticking to defined quality criteria under performance of suitable audits $[4,5,6]$. A higher quality of medical care and a better cost efficiency is supposed to be achieved by certification [4, 7]. It was shown that certification in an university setup led to no essential improvement in quality of care, but to considerable increase on personnel and financial expenditure [2, 4].

This study investigates, if certification leads to improvement of quality or complication rate in total hip arthroplasty (THA) in a high-volume non-academic general hospital.

\section{MATERial AND Method}

A retrospective study was conducted comparing the first 100 THA one year before certification according to EndoCert (Group I) with the first 100 THA one year thereafter (group II).

At our institution 250-300 primary THA are performed per year. Certification was established in June 2014. A consecutive series of the first 100 primary total hip replacement surgery performed in the year 2013 (group I) and 2015 (group II) were included.

Data was retrieved from patient charts and rehabilitation center summary reports under special consideration of quality parameters according to EndoCert [5].

These were presence of pre- and postoperative $\mathrm{X}$-rays, presurgical THA templating, surgery time, complications (such as periprosthetic infection, dislocation, trochanter fracture, periprosthetic fracture, thrombosis \& embolism, mortality, neurological complications), patient's satisfaction with inpatient treatment and acetabular inclination in the X-ray.
In group I, 19 Quadra stems (Medacta, Castel San Pietro, Switzerland), 53 Spotorno stems (Zimmer, Warsaw, USA), 17 Fitmore stems (Zimmer, Warsaw, USA) and 11 cemented Mueller straight stems (Zimmer, Warsaw, USA) were implanted. In group II, 16 Quadra stems (Medacta, Castel San Pietro, Switzerland), 43 Spotorno stems (company Zimmer, Warsaw, USA), 21 Fitmore stems (Zimmer, Warsaw, USA) and 20 cemented Mueller straight stems (Zimmer, Warsaw, USA) were implanted.

Seventy-seven Allofit (company Zimmer, Warsaw, USA) and 23 Versafit (Medacta, Castel San Pietro, Switzerland) cups were used in group I. In group II, there were 83 Allofit (Zimmer, Warsaw, USA) and 17 Versafit (Medacta, Castel San Pietro, Switzerland).

In 81 cases ceramic on XPE Polyethylene articulations and in 19 cases ceramic on ceramic articulations were used. In group I, there were 39 men and 61 women, in group II 40 were men and 60 women. In group I 59 right and 41 left hips were operated, in group II there were 44 right hips und 56 left hips. In group I the average age at the operation time was 67. 5 years (min: 44, max. : 86), in group II 67,6 years (min: 44, max.: 87). In group I the average BMI was 28.8 (min: 19, max.: 50), in group II 27,4 (min: 18, max. : 52). The acetabular inclination was measured in the X-ray picture. The statistical evaluation was conducted with the programme SPSS 22. 0. The Kolmogorow Smirnow test, the Mann-Whitney $\mathrm{U}$ test and Chi-square - test were used.

\section{Results}

Pre- and postoperative $\mathrm{x}$-rays were made in both groups in $100 \%$ of the cases $(p>0.05)$. Preoperative templating was performed in both groups in $100 \%$ of the cases ( $p>0.05$ ). The complications are given in table 1. No statistically significant differences were found in the number of total complications. In each group one case of infection was reported $(1 \%)(\mathrm{p}>0$. $05)$ In group I dislocation was reported twice, whereas in group II no dislocation was reported (p>0.05) A periprosthestic fracture occured twice in each group ( $>0.05$ ). In group I no thrombosis / embolism was to be registered, in group II once ( $p>0.05)$. The average surgery time in group I was 74 minutes (min: 35 , max.: 175 ) and in group II 70 minutes (min: 30 , max. : 120) ( $>$; 0.05). In group I surgery time was longer than 90 mins in 19 out of 100 cases (19\%), whereas in group II it was in 13 out of 
100 cases $(13 \%)(\mathrm{p}<0.001)$. The cup inclination in group I was on an average $40.1^{\circ}$ (min: 30 , max: 65), while in group II it was $40.7^{\circ}$ (min: 30, max.: 52) ( $>0.05)$. In group I there were two cases with inclination $>50^{\circ}$, while in group II there was one case (p>0.05). The rate of documented survey of patient's satisfaction rate improved from $67 \%$ in group I to $97 \%$ in group II $(\mathrm{p}<0,001)$.

Table1. List of complications

\begin{tabular}{|l|l|l|}
\hline Complication & Group I & Group II \\
\hline $\begin{array}{l}\text { Periprosthetic joint } \\
\text { infection }\end{array}$ & 1 & 1 \\
\hline Dislocation & 2 & 0 \\
\hline $\begin{array}{l}\text { Periprosthetic } \\
\text { fracture }\end{array}$ & 2 & 2 \\
\hline Thrombembolism & 0 & 1 \\
\hline
\end{tabular}

\section{DISCUSSION}

For years the quality control has been conducted in the medical centres through the BQS institute of quality and patient's security and currently through the institute of applied quality support and research in the health service (AQUA) [2]. Up to now there are only two works dealing with the effects of an EPZ, which, nevertheless come to much different results. Both works [2, 4] were carried out in university hospitals. Hence, the aim of our work was to examine changes in the complication frequency and the quality indicators in the hip endoprosthesis by the establishment of an endoprosthesesis centere (EPZ) in a regional hospital.

Von Lewinski et al [4] could show in her study that in her EPZ good results were achieved with the achievement of target value. By complying consistently with the standards of treatment within the scope of the establishment of the EPZ the relative frequency of the complications could be reduced further, in particular the periprosthetic infections in the primary endoprosthesis. On the hip endoprosthetic, an improvement of the results regarding the rise of the process quality could be proved significantly [4]. We could prove no reduction of the complications in the current study.

Presurgical and postal-surgical X-ray were performed in group I as well as group II in $100 \%$ of the cases. The presurgical prosthesis planning took place in group I as well as group II in $100 \%$ of the cases. In that way we fulfilled the requirements of EndoCert ${ }^{\circledR}$ before the introduction of the EPZ.
Regarding periprosthetic infection, dislocation and periprosthetic fracture we were at the required level of EndoCert $®$ in group I as well as group II. This may be due to the fact that back then we held morbidity conferences regularly which is, since the introduction of the EPZ, now called complication discussion. One of the shortcoming of the study could be the potential occurrence of Recall-Bias during the retrospective analysis because of the possibility of incomplete documentation of the complications in group I as well as group II.

At some level our results coincide with a recent study of Weber et al [2] who could show that no significant improvement could be achieved on the quality of the results by the certification to EndoCert on the available number of patients in his endoprosthetic centre of the maximum care (EPZ Max) in an university hospital. A weakness of our work certainly lies in the lower number of included patients in our study, however, our result shows a clear trend.

In our hospital process sequence were already documented and decided according to the DIN ISO certification of the orthopaedic department before the establishment of an EPZ.

Other elements were inserted by EndoCert ${ }^{\circledR}$ like patient's questioning, documentation of the waiting period of the patients with an appointment and documentation of the time for an appointment and more. The collection of these data is time consuming and it remains to be seen, to what extent this affects the service quality positively. Those, from Von Lewinski et al. [4] proposed, advantages of the improved perception of the centres by the patients is, in our opinion, a pure advertising factor which deals only little with quality. Considering the inflationary rise in number of EPZ and Max EPZ this advantage is invalid.

We agree to Weber et al. [2] that the examination of the result quality requires a huge personnel as well as financial expenditure.

On average costs for our Institution for the yearly certification according to EndoCert are about 5000 euros and an additional annual fee of 3333 euros to ERPD. The extra documentation for the EPZ requires about 4 days per month (28-32 hours per month).

The average duration of a surgery also showed no significant differences. If one look straight at the quality indicator like number of the Surgeries with duration> $90 \mathrm{~min}$, there was 
indeed a statistically significant difference. We could lower the numbers from $19 \%$ to $13 \%$. However these numbers are still clearly far away from the demands of EndoCert.

Before EPZ, the average surgery time was 82,9 minutes. There were surgeries lasting over 100 minutes, although not by much. The usual reason was the longer suture time required by the junior/assistant surgeons. With implementation of EPZ, surgeons are encouraged to perform the skin closure themselves. Moreover, it encourages the junior surgeons to engage themselves more with the surgical steps and surgical instruments in advance, which leads to better understanding and coordination during the surgery. Furthermore, temporarily banning those junior surgeons with repeated longer surgical time has proven to be motivational.

The Survey of Weber et al. [2] comes to the similar result, which showed the numbers to be 31.6 and $29.8 \%$ for a university setting respectively. In 2015 Von Lewinski et al. [4] published a time excess in $24 \%$ of the cases for the primary total hip replacement surgery which corresponds to our findings.

Both of those cited studies were carried out in university facilities. The authors discuss rightly that the training/education aspect accounts for the longer surgery times as is the case in our hospital as well. In our experience the affiliated doctors and general practitioner are "taking away" all those "simple" and "fast" cases, leaving hospitals specially the university hospitals with time consuming complicated ones. So, in our opinion this aspect should be considered in the EndoCert ${ }^{\circledR}$ quality indicator.

Politically, a proof of the quality in medical care is desired and obligatory [2].

The current study shows that only few improvements has been achieved by implementation of EPZ. Although, one has to say that these results cannot be passed on to other Institutions without any limitations. The effects of the required formal reduction of QM measures in the EPZ system may not have been so clear in their own analysis, since several elements of quality assurance (indication discussions, complication discussions, etc.) have already existed through the previously existing QM system. Furthermore, it deals with a small department with constant and experienced main operators. Another drawback of the study is the retrospective evaluation of the data. For these reasons, the transferability and meaningfulness of the data as a whole is clearly limited, but a tendency can be deduced and should give reason to rethink and dynamically develop the current system of EPZ.

\section{CONCLuSiON}

In Conclusion, it can be stated that the introduction of an endoprosthesis centre (EPZ) has led to limited positive changes in a general hospital. At most the quality indicator "Surgery time > $90 \mathrm{~min}$ " has improved significantly. Nevertheless, the considerable personnel and financial expenditure does not justify those minimal improvements. In our opinion, a quality management system is necessary. It should be properly examined, whether or not other certification systems like DIN EN ISO9001:2015 are enough. At that moment we recommend not to follow this german concept.

\section{CONFLICT OF INTEREST}

The authors declare that they have no conflict of interest.

\section{FUNDING}

There is no funding source.

\section{ETHICAL APPROVAL}

This article does not contain any studies with human participants or animals performed by any of the authors.

\section{LITERATURE}

[1] I.D. Learmonth, C. Young, C. Rorabeck. The operation of the century: total hip replacement. Lancet 370 (2007) 1508-1519.

[2] P. Weber, A.C. Paulus, D. Hallmen, A. Steinbrück, F. Schmidutz, V. Jansson. Does the certification according to EndoCert lead to a better quality of treatment? Orthopäde. 46 (2017) 78 - 88.

[3] S. Mitsuyasu, A. Hagihara, H. Horiguchi, K. Nobutomo . Relationship between total arthroplasty case volume and patient outcome in an acute care payment system in Japan. J Arthroplasty 21 (2006) 656 - 663.

[4] G. von Lewinski, T. Floerkemeier, S. Budde, U. Fuhrmann, M. Schwarze, h. Windhagen, K. Radtke. Experience in establishing a certified endoprosthesis center. Orthopäde. 44 (2015) $193-202$.

[5] H. Haas, J. Grifka, K.P. Günther, H.D. Heller, F.U. Niethard, H. Windhagen, M. Ebner, W. Mittelmeier W. EndoCert $₫$ Zertifizierung von Endoprothetischen Versorgungszentren in 
Does Certification of an Arthroplasty Center according to Endocert Improve Quality or Complication Rate in Total Hip Arthroplasty in a General Hospital? Don't Follow Germany?

Deutschland. Georg Thieme Verlag Stuttgart, New York 2013.

[6] H. Haas, W. Mittelmeier. Qualitätssicherung in der Endoprothtik. Deutsches Ärzteblatt 100 (2013) $318-319$.
[7] M. Eberlein-Gonska, S. Schellong, M. Baumann. Certified medical centers--A measurable benefit to patients? Z Arztl Fortbild Qualitatssich. 101 (2007) 173 - 179.

Citation: Lohani Bibek, Wolfgang Hönle, Stefan Sesselmann, Alexander Scheller, Philipp Köhl, Alexander Schuh. "Does Certification of an Arthroplasty Center according to Endocert Improve Quality or Complication Rate in Total Hip Arthroplasty in a General Hospital? Don't Follow Germany!’. 2021; 6(1):18-22. DOI:https://doi.org/10.20431/2456-0588.0601005.

Copyright: (C) 2021 Authors. This is an open-access article distributed under the terms of the Creative Commons Attribution License, which permits unrestricted use, distribution, and reproduction in any medium, provided the original author and source are credited. 